\title{
Asymptotic properties of a branching random walk with a random environment in time
}

\author{
Yuejiao Wang ${ }^{\dagger}$ Zaiming Liu ${ }^{\dagger}$ Quansheng Liu ${ }^{\ddagger}$ Yingqiu $\operatorname{Li}^{\S}$
}

\begin{abstract}
We consider a branching random walk in an independent and identically distributed random environment $\xi=\left(\xi_{n}\right)$ indexed by the time. Let $W$ be the limit of the martingale $W_{n}=\int e^{-t x} Z_{n}(d x) / \mathbb{E}_{\xi} \int e^{-t x} Z_{n}(d x)$, with $Z_{n}$ denoting the counting measure of particles of generation $n$, and $\mathbb{E}_{\xi}$ the conditional expectation given the environment $\xi$. We find necessary and sufficient conditions for the existence of quenched moments and weighted moments of $W$, when $W$ is non-degenerate.
\end{abstract}

Key words Branching random walk, random environment, quenched moments, weighted moments

\section{Introduction and main results}

The model of branching random walk has been studied by many authors, see e.g. [7, 8, 19, 31, $28,13]$ and the references therein. A branching random walk with a random environment in time is an important extension in which the offspring distribution of a particle of generation $n$, and the distribution of the displacements of their children, depend on the environment $\xi=(\xi)_{n}$ indexed by the time $n$, cf. e.g. $[9,27,28,29]$.

The model of branching random walk with a random environment in time can be described as follows. As usual, let $\mathbb{N}=\{0,1,2, \cdots\}, \mathbb{N}^{+}=\{1,2, \cdots\}, \mathbb{R}=\{-\infty, \infty\}$ and

$$
U=\bigcup_{n=0}^{\infty}\left(\mathbb{N}^{+}\right)^{n}
$$

be the set of all finite sequences, where $(\mathbb{N})^{+0}=\{\emptyset\}$ contains the null sequence $\emptyset$. Let $\xi=\left(\xi_{n}\right)_{n \geq 0}$ be a sequence of independent and identically distribution random variables taking values in some space $\Theta$; each realization of $\xi_{n}$ corresponds probability distribution $\eta_{n}=\eta\left(\xi_{n}\right)$ on $\mathbb{N} \times \mathbb{R} \times \mathbb{R} \times \cdots$. Here $\xi_{n}$ represents the random environment at time $n$. When the environment sequence $\xi$ is given, the branching random walk starts from an initial particle $\emptyset$ of generation 0 located at the origin $S_{\emptyset}=0 \in \mathbb{R}$. It gives birth to $N_{\emptyset}=N$ children of the first generation whose number and displacements (relative to their parent $\emptyset$ ) $L_{\emptyset i}=L_{i}$ constitute a point process $\left(N ; L_{1}, L_{2}, \cdots\right)$ with distribution $\eta_{0}=\eta\left(\xi_{0}\right)$ on $\mathbb{N} \times \mathbb{R} \times \mathbb{R} \times \cdots$.

\footnotetext{
${ }^{\dagger}$ Central South University, School of Mathematics and Statistics, 410083, Changsha, China. Email: wangyuejiaohujing@163.com (Yuejiao), math_lzm@csu.edu.cn (Zaiming)

${ }^{\ddagger}$ Corresponding author. Univ. Bretagne-Sud, UMR 6205, Laboratoire de Mathématiques et Applications des Mathématiques, F-56000 Vannes, France. Email:quansheng.liu@univ-ubs.fr

${ }^{\S}$ Changsha University of Science and Technology, School of Mathematics and Statistics, 410004, Changsha, China. Email: liyq-2001@163.com
} 
In general, when the environment $\xi$ is given, each particle $u=u_{1} \cdots u_{n}$ of $n$-th generation with position $S_{u}$ gives birth to $N_{u}$ children with displacements $L_{u, i}$, so that the position of the $i$-th child is

$$
S_{u i}=S_{u}+L_{u i}
$$

where $\left(N_{u} ; L_{u 1}, L_{u 2}, \cdots\right)$ has distribution $\eta_{n}=\eta\left(\xi_{n}\right)$ on $\mathbb{N} \times \mathbb{R} \times \mathbb{R} \times \cdots$. Conditioned on the environment $\xi$, all particles behave independently, which means that the family of the random vectors $\left(N_{u} ; L_{u 1}, L_{u 2}, \cdots\right)$, indexed by all finite sequences $u$, are conditionally independent.

Let $\mathbb{T}$ be the Galton-Watson tree with defining elements $\left\{N_{u}: u \in U\right\}:$ (i) $\emptyset \in \mathbb{T}$; (ii) if $u \in \mathbb{T}$, then $u i \in \mathbb{T}$ if only if $1 \leq i \leq N_{u}$; (iii) if $u i \in \mathbb{T}$, then $u \in \mathbb{T}$.

The family $\left\{S_{u}, u \in \mathbb{T}\right\}$ is called a branching random walk with a random environment in time. In the following it will be termed simply as a branching random walk in a random environment.

Let

$$
Z_{n}=\sum_{|u|=n} \delta_{S_{u}}
$$

be the counting measure of particles of generation $n$, so that for a subset $A$ of $\mathbb{R}, Z_{n}(A)$ is the number of particles of generation $n$ located in $A$ :

$$
Z_{n}(A)=\sum_{|u|=n} \delta_{S_{u}}(A)=\sum_{|u|=n} I_{A}\left(S_{u}\right),
$$

where $\delta_{S_{u}}$ denotes the Dirac measure at $S_{u}$ and $I_{A}$ the indicator function of $A$; by convention the summation is over all particles $u$ of generation $n$.

The total probability space on which all the random variables $\xi_{n}$ and $L_{u i},|u|=n \geq 0$ are defined will be denoted by $(\Omega, \mathcal{F}, \mathbb{P})$; the conditional probability given the environment $\xi$ will be denoted by $\mathbb{P}_{\xi}$. Therefore, by definition, for each realization of the environment sequence $\xi$, the random variables $L_{u i}(|u|=n \geq 0, i \geq 1)$ are independent of each other under $\mathbb{P}_{\xi}$. The probability $\mathbb{P}$ is usually called annealed law, while $\mathbb{P}_{\xi}$ is called quenched law. The expectation with respect to $\mathbb{P}$ and $\mathbb{P}_{\xi}$ will be denoted respectively by $\mathbb{E}$ and $\mathbb{E}_{\xi}$.

Fix $t \in \mathbb{R}$. Write

$$
m_{n}(t)=\mathbb{E}_{\xi} \sum_{i=1}^{N_{u}} e^{-t L_{u i}} \text { for }|u|=n,
$$

and assume that $m_{n}(t)<\infty$. We are interested in the Laplace transform of the counting measure $Z_{n}$ and the associated martingale:

$$
\begin{aligned}
& \hat{Z}_{n}(t)=\int e^{-t x} Z_{n}(d x)=\sum_{|u|=n} e^{-t S_{u}}, \quad n \geq 0 \\
& W_{0}(t)=1, \quad W_{n}(t)=\frac{\hat{Z}_{n}(t)}{\mathbb{E}_{\xi} \hat{Z}_{n}(t)}=\frac{1}{\prod_{i=1}^{n-1} m_{i}(t)} \sum_{|u|=n} e^{-t S_{u}} \text { for } n \geq 1 .
\end{aligned}
$$

It is well known that $\left(W_{n}(t)\right)_{n \geq 0}$ is a nonnegative martingale under $\mathbb{P}_{\xi}$ with respect to the filtration

$$
\mathcal{F}_{0}=\sigma(\xi), \mathcal{F}_{n}=\sigma\left(\xi, N_{u}, L_{u 1}, L_{u 2}, \cdots,|u|<n\right) \text { for } n \geq 1
$$


so that the limit $W(t)=\lim _{n \rightarrow \infty} W_{n}(t)$ exists almost surely (a.s.) with $\mathbb{E}_{\xi} W(t) \leq 1$ by Fatou's Lemma. For simplicity, write

$$
W_{n}=W_{n}(t) \text { for } n \geq 0 \text { and } W=W(t) .
$$

The necessary and sufficient conditions for the non-degeneration of $W$ have been known: see Proposition 2.1 below applied to $A_{u i}=e^{-t L_{u i}}$. The existence of annealed moments and weighted moments of $Y$ have been studied in [22], see Theorems 2.1 and 2.2 cited below. Here we are interested in the quenched moments and weighted moments of $W$, when $W$ is non-degenerate.

We first consider the existence of the quenched moments of $W$.

Theorem 1.1 Let $\alpha>1$, and assume that $\mathbb{P}(W=0)<1$.

(i) If $\mathbb{E} \log ^{+} \mathbb{E}_{\xi} W_{1}^{\alpha}<\infty$ and $\mathbb{E} \log \frac{m_{0}(\alpha t)}{m_{0}^{\alpha}(t)} \in(-\infty, 0)$, then $\mathbb{E}_{\xi} W^{\alpha}<\infty$ a.s..

(ii) If $\mathbb{E} \log \frac{m_{0}(\alpha t)}{m_{0}^{\alpha}(t)} \in(-\infty, 0)$ and $\mathbb{E}_{\xi} W^{\alpha}<\infty$ a.s., then $\mathbb{E} \log ^{+} \mathbb{E}_{\xi} W_{1}^{\alpha}<\infty$.

(iii) If $\mathbb{E}\left(\log ^{-} \mathbb{E}_{\xi} W_{1}\right)^{2+\varepsilon}<\infty$ for some $\varepsilon>0, \mathbb{E} \log ^{+} \mathbb{E}_{\xi} W_{1}^{\alpha}<\infty, \mathbb{E}\left(\log \frac{m_{0}(\alpha t)}{m_{0}^{\alpha}(t)}\right)^{2}<\infty$ and $\mathbb{E}_{\xi} W^{\alpha}<\infty$ a.s., then $\mathbb{E} \log \frac{m_{0}(\alpha t)}{m_{0}^{\alpha}(t)}<0$.

Part (i) gives sufficient conditions for the existence of quenched moments $\mathbb{E}_{\xi} W^{\alpha}$, while parts (ii) and (iii) show that these conditions are also necessary under some additional assumptions.

Recall that a positive and measurable function $l$ is defined on $[0, \infty)$ is called slowly varying at $\infty$ if $\lim _{x \rightarrow \infty} \frac{l(\lambda x)}{l(x)}=1$ for all $\lambda>0$. (Throughout this paper, the term "positive" is used in the wide sense.) By the representation theorem (see [12], Theorem 1.3.1), any function $l$ slowly varying at $\infty$ is of the form

$$
l(x)=c(x) \exp \left(\int_{a_{0}}^{x} \frac{\varepsilon(t)}{t} d t\right), \quad x>a_{0},
$$

where $a_{0}>0, c(\cdot)$ and $\varepsilon(\cdot)$ are measurable with $c(x) \rightarrow c$ for some constant $c \in(0, \infty)$ and $\varepsilon(x) \rightarrow 0$ as $x \rightarrow \infty$. Moreover, it is known that any slowly varying function $l$ posses a smoothed version $l_{1}$ in the sense that $l(x) \sim l_{1}(x)$ as $x \rightarrow \infty$, with $l_{1}$ of the form

$$
l_{1}(x)=c \exp \left(\int_{a_{0}}^{x} \frac{\varepsilon_{1}(t)}{t} d t\right), \quad x>a_{0},
$$

with $\varepsilon_{1}$ infinitely differentiable on $\left(a_{0}, \infty\right)$ and $\lim _{x \rightarrow \infty} \varepsilon_{1}(x)=0$ (see [12], Theorem 1.3.3). The value of $a_{0}$ and those of $l(x)$ on $\left[0, a_{0}\right]$ will not be important. For convenience, we often take $a_{0}=1$. Notice also that the function $c(\cdot)$ in the representation of $l(\cdot)$ has no influence on the finiteness of moments of $W$ of the form $\mathbb{E}_{\xi} W^{\alpha} l(W)$, so that we can suppose without loss of generality that $c(x)=1$. Moreover, by choosing a smoothed version if necessary, we can suppose that the function $\varepsilon$ in the representation form (1.1) is infinitely differentiable.

We next give a description of the quenched weighted moments of $W$. Write $W^{*}=$ $\sup _{n \geq 1} W_{n}$. 
Theorem 1.2 Assume that $\mathbb{P}(W=0)<1$ and $\mathbb{E} \log \frac{m_{0}(\alpha t)}{m_{0}^{\alpha}(t)} \in(-\infty, 0)$. Let $l(x)$ be a function slowly varying at $\infty$ and $\phi(x)=x^{\alpha} l(x)$ with $\alpha>1$. Then the following assertions are equivalent:

(i) $\mathbb{E} \log ^{+} \mathbb{E}_{\xi} \phi\left(W_{1}\right)<\infty$; (ii) $\mathbb{E}_{\xi} \phi(W)<\infty$ a.s.; (iii) $\mathbb{E}_{\xi} \phi\left(W^{*}\right)<\infty$ a.s. .

The rest of the paper is organized as follows. In Section 2 we describe the model of Mandelbrot's martingale in a random environment, and state for this martingale a variante of Theorems 1.1 and 1.2: see Theorems 2.3 and 2.4, which imply Theorems 1.1 and 1.2. In Section 3, we introduce some lemmas in order to prove our main results. In Sections 4 and 5 , we give respectively the proof of Theorems 2.3 and 2.4 .

\section{Mandelbrot's martingale in a random environment}

The theorems stated in Section 1 will be proved for a slightly different but essentially equivalent model, i.e., for Mandelbrot's martingale in a random environment. This model is described as follows. Let $\xi=\left(\xi_{n}\right)_{n \geq 0}$ be a sequence of independent and identically distribution random variables taking values in some space $\Theta$. Suppose that when the environment $\xi$ is given, $\left\{\left(N_{u}, A_{u 1}, A_{u 2}, \cdots\right): u \in U\right\}$ is a sequence of independent random variables with values in $\mathbb{N} \times \mathbb{R}_{+}^{\mathbb{N}^{+}}$, where $\mathbb{R}_{+}=[0, \infty)$, defined on some probability space $\left(\Gamma, \mathbb{P}_{\xi}\right)$; each $\left(N_{u}, A_{u 1}, A_{u 2}, \cdots\right)$ has distribution $\eta\left(\xi_{n}\right)$ for $|u|=n$. For simplicity, we write $\left(N, A_{1}, A_{2}, \cdots\right)$ for $\left(N_{\emptyset}, A_{\emptyset 1}, A_{\emptyset 2}, \cdots\right)$.

Set

$$
\begin{aligned}
& m_{n}=\mathbb{E}_{\xi} \sum_{i=1}^{N_{u}} A_{u i}, \text { where }|u|=n, n \geq 0 . \\
& X_{\emptyset}=1, X_{u}=\frac{A_{u_{1}} A_{u_{1} u_{2}} \cdots A_{u_{1} \cdots u_{n}}}{\prod_{i=0}^{n-1} m_{i}}, \text { if } u=u_{1} \cdots u_{n} \in U, \text { for } n \geq 1 . \\
& Y_{0}=1 \text { and } Y_{n}=\sum_{|u|=n} X_{u}, \text { for } n \geq 1 .
\end{aligned}
$$

Then, under $\mathbb{P}_{\xi}$, the sequence $\left(Y_{n}\right)_{n \geq 0}$ forms a nonnegative martingale with respect to the filtration

$$
\mathcal{G}_{0}=\sigma(\xi) \text { and } \mathcal{G}_{n}=\sigma\left(\xi, N_{u}, A_{u 1}, A_{u 2}, \cdots,|u|<n\right) \text { for } n \geq 1 .
$$

It follows that $\left(Y_{n}, \mathcal{G}_{n}\right)$ is also a martingale under $\mathbb{P}$. The martingale $\left(Y_{n}\right)$ is called Mandelbrot's martingale in a random environment. Notice that the martingale $\left(W_{n}\right)$ for the branching random walk $\left(S_{u}, u \in \mathbb{T}\right)$ introduced in Section 1 is just Mandelbrot's martingale $\left(Y_{n}\right)$ with $A_{u i}=e^{-t L_{u i}}$ for $|u|=n \geq 0$.

Let

$$
Y=\lim _{n \rightarrow \infty} Y_{n} \text { and } Y^{*}=\sup _{n \geq 0} Y_{n}
$$

where the limit exists a.s. by the martingale convergence theorem, and $\mathbb{E}_{\xi} Y \leq 1$ by Fatou's lemma.

We can image that each node of the tree $\mathbb{T}$ is marked with the vector $\left(N_{u}, A_{u 1}, A_{u 2}, \cdots\right)$, $A_{u i}$ being associated with the edge $(u, u i)$ linking $u$ and $u i$ for $u \in \mathbb{T}$ and $1 \leq i \leq N_{u}$; 
the values of $A_{u i}$ for $i>N_{u}$ are of no influence for our results and will be taken as 0 for convenience. So the model is also called weighted branching process in a random environment. See for example [20].

Remark 2.1 If $A_{u} \equiv 1$ for all $u$, then $\left(Y_{n}\right)$ becomes the natural martingale of the branching process in a random environment, studied by many authors: see for example [1, 2, 3].

We are interested in the asymptotic properties of Mandelbrot's martingale in a random environment. For the existence of moments of $Y$ in the Mandelbrot's martingale, Liu [26] proved that $\mathbb{E} Y^{\alpha}<\infty$ if and only if $\mathbb{E} Y_{1}^{\alpha}<\infty$ and $\mathbb{E} \sum_{i=1}^{N} A_{i}^{\alpha}<1$. In this paper, we extend this result to the Mandelbrot's martingale in a random environment for the quenched law, for which we will show that the existence condition is quite different to the annealed case.

Another interest is the existence of the weighted moments of $Y$ of the form $\mathbb{E}_{\xi} Y^{\alpha} l(Y)$, where $\alpha>1$ and $l$ is a positive function slowly varying at $\infty$. For a Galton-Watson process, Bingham and Doney [10] showed that when $\alpha>1$ is not an integer, $\mathbb{E} Y^{\alpha} l(Y)<\infty$ if and only if $\mathbb{E} Y_{1}^{\alpha} l\left(Y_{1}\right)<\infty$. Alsmeyer and Rösler [4] proved that the same result remains true for all non-dyadic integer $\alpha>1$ (not of the form $2^{k}$ for some integer $k \geq 0$ ). Liang and Liu [23] proved that the result holds true for all $\alpha>1$. For the Mandelbrot's martingale, Alsmeyer and Kuhlbusch [5] showed that when $\alpha \in\left\{2^{n}: n \geq 1\right\}, \mathbb{E}^{\alpha} l(Y)<\infty$ if and only if $\mathbb{E} Y_{1}^{\alpha} l\left(Y_{1}\right)<\infty$. Liang and Liu [25] proved that the same result remains true for all $\alpha>1$ and $l$ is a positive function slowly varying at $\infty$. In [22], this result was further extended to the Mandelbrot's martingale in a random environment for the annealed weighted moments of $Y$ of the form $\mathbb{E} Y^{\alpha} l(Y)$. In this paper, we consider the quenched weighted moments of $Y$ for the Mandelbrot's martingale with a random environment; we will see that the existence condition is quite different to the annealed case.

For any $x \geq 0$, write

$$
\begin{aligned}
\rho_{\xi_{n}}(x) & =\mathbb{E} \xi \sum_{i=1}^{N_{u}} \frac{A_{u i}^{x}}{m_{n}^{x}} \text { for }|u|=n, n \geq 0 . \\
\rho(x) & =\mathbb{E} \rho_{\xi_{0}}(x)=\mathbb{E} \sum_{i=1}^{N} \frac{A_{i}^{x}}{m_{0}^{x}} \quad \text { and } \\
\rho^{\prime}(x) & =\mathbb{E} \sum_{i=1}^{N} \frac{A_{i}^{x}}{m_{0}^{x}} \log \frac{A_{i}}{m_{0}}
\end{aligned}
$$

if the expression is well defined (with value in $[-\infty, \infty]$ ). We mention that here $\rho^{\prime}(x)$ is a notation, which coincides with the derivative of $\rho(\cdot)$ at $x$ under natural regularity conditions.

For the non-degeneration of $Y$, a necessary and sufficient condition was shown by Biggins and Kyprianou(2004,Theorem 7.1) and Kuhlbusch (2004,Theorem 2.5).

Proposition 2.1 (Non-degeneration [9],[20]) Assume that $\rho^{\prime}(1)$ is well-defined with value in $[-\infty, \infty)$. Then the following assertions are equivalent:

(i) $\rho^{\prime}(1)<0$ and $\mathbb{E} Y_{1} \log ^{+} Y_{1}<\infty$.

(ii) $\mathbb{E} Y=1$. 
(iii) $\mathbb{P}(Y=0)<1$.

Necessary and sufficient conditions for the existence of annealed moments and weighted moments of $Y$ have been known. Let us recall them in the following two theorems.

Theorem 2.1 ([22]) Assume $\mathbb{P}(Y=0)<1$. For $\alpha>1$, the following assertion are equivalent:

(i) $\mathbb{E} Y_{1}^{\alpha}<\infty$ and $\rho(\alpha)<1$;

(ii) $\mathbb{E} Y^{*}<\infty$;

(iii) $0<\mathbb{E} Y^{\alpha}<\infty$.

Theorem 2.2 ([22]) Assume that $\mathbb{P}(Y=0)<1$ and $\rho(\alpha)<1$ for $\alpha>1$. Let $l:[0, \infty) \rightarrow$ $[0, \infty)$ be a function slowly varying at $\infty$. Then the following assertions are equivalent:

(i) $\mathbb{E} Y_{1}^{\alpha} l\left(Y_{1}\right)<\infty$;

(ii) $\mathbb{E} Y^{* \alpha} l\left(Y^{*}\right)<\infty$;

(iii) $0<\mathbb{E} Y^{\alpha} l(Y)<\infty$,

Here we are interested in the quenched moments and weighted moments of $Y$, when $Y$ is non-degenerate. We first consider the quenched moments.

Theorem 2.3 Let $\alpha>1$, and assume that $\mathbb{P}(Y=0)<1$.

(i) If $\mathbb{E} \log ^{+} \mathbb{E}_{\xi} Y_{1}^{\alpha}<\infty$ and $\mathbb{E} \log \rho_{\xi_{0}}(\alpha) \in(-\infty, 0)$, then $\mathbb{E}_{\xi} Y^{\alpha}<\infty$ a.s..

(ii) If $\mathbb{E} \log \rho_{\xi_{0}}(\alpha) \in(-\infty, 0)$ and $\mathbb{E}_{\xi} Y^{\alpha}<\infty$ a.s., then $\mathbb{E} \log ^{+} \mathbb{E}_{\xi} Y_{1}^{\alpha}<\infty$.

(iii) If $\mathbb{E}\left(\log ^{-} \mathbb{E}_{\xi} Y_{1}\right)^{2+\varepsilon}<\infty$ for some $\varepsilon>0$, $\mathbb{E} \log ^{+} \mathbb{E}_{\xi} Y_{1}^{\alpha}<\infty$, $\mathbb{E} \log ^{2} \rho_{\xi_{0}}(\alpha)<\infty$ and $\mathbb{E}_{\xi} Y^{\alpha}<\infty$ a.s., then $\mathbb{E} \log \rho_{\xi_{0}}(\alpha)<0$.

Part (i) gives sufficient conditions for the existence of quenched moments $\mathbb{E}_{\xi} Y^{\alpha}$, while parts (ii) and (iii) show that these conditions are also necessary under some additional assumptions.

Notice that since $\left(Y_{n}\right)$ is a nonnegative martingale under $\mathbb{P}_{\xi}$, the existence of quenched moments $\mathbb{E}_{\xi} Y^{\alpha}$ is equivalent to the convergence in $L^{\alpha}$ under $\mathbb{P}_{\xi}$.

We next consider the existence of the quenched weighted moments of $Y$.

Theorem 2.4 Assume that $\mathbb{P}(Y=0)<1$ and $\mathbb{E} \log \rho_{\xi_{0}}(\alpha) \in(-\infty, 0)$. Let $l(\cdot)$ be a function slowly varying at $\infty$ and $\phi(x)=x^{\alpha} l(x)$ with $\alpha>1$. Then the following assertions are equivalent:

(i) $\mathbb{E} \log ^{+} \mathbb{E}_{\xi} \phi\left(Y_{1}\right)<\infty$; (ii) $\mathbb{E}_{\xi} \phi(Y)<\infty$ a.s.; (iii) $\mathbb{E}_{\xi} \phi\left(Y^{*}\right)<\infty$ a.s..

Clearly, Theorems 1.1 and 1.2 come from Theorems 2.3 and 2.4 with $A_{u i}=e^{-t L_{u i}}$.

\section{Preliminary lemmas}

For the proof of our main results Theorems 1.1 and 1.2, we will use the following Lemmas.

Lemma 3.1 Let $\left(\alpha_{n}, \beta_{n}\right)_{n \geq 0}$ be a stationary and ergodic sequence of non-negative random variables. 
(1) If $\mathbb{E} \log \alpha_{0}<0$ and $\mathbb{E} \log ^{+} \beta_{0}<\infty$, then

$$
\sum_{n=0}^{\infty} \alpha_{0} \cdots \alpha_{n-1} \beta_{n}<\infty \quad \text { a.s.. }
$$

(2) Conversely, we have:

(a) if $\mathbb{E} \log \alpha_{0} \in(-\infty, 0)$ and $\left(\alpha_{n}, \beta_{n}\right)_{n \geq 0}$ is i.i.d., then (3.1) implies that $\mathbb{E} \log ^{+} \beta_{0}<\infty$;

(b) if $\mathbb{E}\left|\log \beta_{0}\right|<\infty$, then (3.1) implies that $\mathbb{E} \log \alpha_{0} \leq 0$;

(c) if $\mathbb{E}\left|\log \beta_{0}\right|<\infty$ and $\mathbb{E}\left(\log ^{-} \beta_{0}\right)^{2+\varepsilon}<\infty$ for some $\varepsilon>0$, then (3.1) implies that

$\mathbb{E} \log \alpha_{0}<0$, provided that $\mathbb{E}\left(\log \alpha_{0}\right)^{2}<\infty$ and that $\left(\alpha_{n}\right)_{n}$ is i.i.d.

For Part (1) and the first two conclusions of Part (2), see ([18], Lemma 3.1). See also [15] and [21] for a discussion about the convergence of the series (3.1). Below we give a proof of the last conclusion of Part (2).

Proof of Lemma 3.1. As mentioned above, we only need to prove (c) of Part (2). By (b) of Part (2), we know that $\mathbb{E} \log \alpha_{0} \leq 0$. Assume that $\mathbb{E} \log \alpha_{0}=0$. Since $\mathbb{E}\left(\log ^{-} \beta_{0}\right)^{2+\varepsilon}<\infty$, we have for any constant $c>0$,

$$
\begin{aligned}
& \sum_{n=3}^{\infty} \mathbb{P}\left(\beta_{n}<\exp (-c \sqrt{n \log \log n})\right) \\
= & \sum_{n=3}^{\infty} \mathbb{P}\left(\log ^{-} \beta_{n}>c \sqrt{n \log \log n}\right) \\
\leq & \sum_{n=3}^{\infty} \frac{\mathbb{E}\left(\log ^{-} \beta_{0}\right)^{2+\varepsilon}}{(c \sqrt{n \log \log n})^{2+\varepsilon}}<\infty .
\end{aligned}
$$

So by Borel-Cantelli's lemma, we have a.s.

$$
\beta_{n} \geq \exp (-c \sqrt{n \log \log n}) \text { for all } n \text { large enough. }
$$

On the other hand, since $\sigma^{2}:=\mathbb{E}\left(\log \alpha_{0}\right)^{2}<\infty$, by the law of iterated logarithm, we have

$$
\limsup _{n \rightarrow \infty} \frac{\sum_{i=0}^{n-1} \log \alpha_{i}}{\sqrt{\sigma^{2} n \log \log n}}=1 \quad \text { a.s.. }
$$

Therefore, choosing $0<c<\frac{\sigma}{2}$, we see that a.s., for infinitely many $n$,

$$
\alpha_{0} \alpha_{1} \cdots \alpha_{n-1} \beta_{n} \geq \exp \left(\left(\frac{\sigma}{2}-c\right) \sqrt{n \log \log n}\right) \rightarrow \infty,
$$

which is a contradiction with (3.1). This shows that $\mathbb{E} \log \alpha_{0}<0$.

For the proof of our main results, we will use the Burkholder-Davis-Gundy(BDG) inequalities that we are going to state in the following lemma. For a martingale sequence $\left\{\left(f_{n}, \mathcal{G}_{n}\right): n \geq 1\right\}$ defined on some probability space $(\Omega, \mathcal{G}, \mathbb{P})$, set $f_{0}=0, \mathcal{G}_{0}=\{\emptyset, \Omega\}$, $d_{n}=f_{n}-f_{n-1}$ for $n \geq 1$,

$$
f^{*}=\sup _{n \geq 1}\left|f_{n}\right| \quad \text { and } \quad d^{*}=\sup _{n \geq 1}\left|d_{n}\right| .
$$


Lemma 3.2 ([14],Theorem 2) Let $\Phi:[0, \infty) \rightarrow[0, \infty)$ be an increasing and continuous function with $\Phi(0)=0$ and $\Phi(2 \lambda) \leq c \Phi(\lambda)$ for some $c \in(0, \infty)$ and all $\lambda>0$.

(i) For every $\beta \in(1,2]$, there exists a constant $B=B_{c, \beta} \in(0, \infty)$ depending only on $c$ and $\beta$ such that for any martingale $\left\{\left(f_{n}, \mathcal{G}_{n}\right): n \geq 1\right\}$, we have

$$
\mathbb{E} \Phi\left(f^{*}\right) \leq B \mathbb{E} \Phi(s(\beta))+B \mathbb{E} \Phi\left(d^{*}\right) \text { with } \quad s(\beta)=\left(\sum_{n=1}^{\infty} \mathbb{E}\left(\left|d_{n}\right|^{\beta} \mid \mathcal{G}_{n-1}\right)\right)^{1 / \beta}
$$

and

$$
\mathbb{E} \Phi\left(f^{*}\right) \leq B \mathbb{E} \Phi(s(\beta))+B \sum_{n=1}^{\infty} \mathbb{E} \Phi\left(\left|d_{n}\right|\right)
$$

(ii) If $\Phi$ is convex on $[0, \infty)$, then there exist constants $A=A_{c} \in(0, \infty)$ and $B=B_{c} \in$ $(0, \infty)$, depending only on $c$, such that for any martingale $\left\{\left(f_{n}, \mathcal{G}_{n}\right): n \geq 1\right\}$, we have

$$
A \mathbb{E} \Phi(S) \leq \mathbb{E} \Phi\left(f^{*}\right) \leq B \mathbb{E} \Phi(S), \quad \text { where } S=\left(\sum_{n=1}^{\infty} d_{n}^{2}\right)^{1 / 2}
$$

moreover, for any $\beta \in(0,2]$,

$$
\mathbb{E} \Phi\left(f^{*}\right) \leq B \mathbb{E} \Phi(S(\beta)), \text { where } S(\beta)=\left(\sum_{n=1}^{\infty}\left|d_{n}\right|^{\beta}\right)^{1 / \beta} .
$$

If, additionally, for some $\beta \in(0,2]$ the function $\Phi_{1 / \beta}(x)=\Phi\left(x^{1 / \beta}\right)$ is subadditive on $[0, \infty)$, then

$$
\mathbb{E} \Phi\left(f^{*}\right) \leq B \sum_{n=1}^{\infty} \mathbb{E} \Phi\left(\left|d_{n}\right|\right)
$$

\section{Proof of Theorem 2.3}

Without loss of generality, we assume that $m_{n}=1$ a.s.. Otherwise we can consider $\tilde{A}_{u i}:=$ $A_{u i} / m_{n}$ instead of $A_{u i}$, for $|u|=n, n \geq 0$.

For $x>0$ and $n \geq 0$, write

$$
Y_{n}(x):=\sum_{|u|=n} X_{u}^{x} \text { and } P_{n}(x):=\mathbb{E}_{\xi} \sum_{|u|=n} X_{u}^{x}=\rho_{\xi_{0}}(x) \cdots \rho_{\xi_{n-1}}(x) .
$$

Obviously, $Y_{n}=Y_{n}(1)$ and $\mathbb{E}_{\xi} Y_{1}(x)=\mathbb{E}_{\xi} \sum_{i=1}^{N} A_{i}^{x}=\rho_{\xi_{0}}(x)$. Let

$$
Y_{n+1}-Y_{n}=\sum_{|u|=n} X_{u}\left(Y_{1}(u)-1\right)
$$

where $Y_{1}(u)=\sum_{|v|=1} A_{u v}, \mathbb{P}_{\xi}\left(Y_{1}(u) \in \cdot\right)=\mathbb{P}_{T^{n} \xi}\left(Y_{1} \in \cdot\right)$ for $|u|=n$. Let

$$
D_{n}=\sup _{k \geq 1} A_{u k},|u|=n \text { and } D_{n}^{*}=\sup _{|u|=n} X_{u} \leq D_{0} \cdots D_{n-1} .
$$


Proof of Theorem 2.3 Notice that since $\left(Y_{n}\right)$ is a martingale under $\mathbb{P}_{\xi}, \mathbb{E}_{\xi} Y^{\alpha}<\infty$ a.s. is equivalent to $\sup _{n} \mathbb{E}_{\xi} Y_{n}^{\alpha}<\infty$ a.s.. Obviously, the condition $\sup _{n} \mathbb{E}_{\xi} Y_{n}^{\alpha}<\infty$ a.s. is equivalent to $\sup _{n} \mathbb{E}_{\xi}\left|Y_{n}-1\right|^{\alpha}<\infty$ a.s..

We first prove part (i). Suppose that $\mathbb{E} \log ^{+} \mathbb{E}_{\xi} Y_{1}^{\alpha}<\infty$. For $\alpha \in(1,2]$, the result has been proved by [17]. So we assume $\alpha>2$. Using BDG-inequality, we have

$$
\sup _{n} \mathbb{E}_{\xi}\left|Y_{n}-1\right|^{\alpha} \leq C \mathbb{E}_{\xi}\left|\sum_{n=0}^{\infty}\left(Y_{n+1}-Y_{n}\right)^{2}\right|^{\frac{\alpha}{2}} .
$$

Since $\left(\mathbb{E}_{\xi}\left|\sum_{n=0}^{\infty}\left(Y_{n+1}-Y_{n}\right)^{2}\right|^{\frac{\alpha}{2}}\right)^{\frac{2}{\alpha}} \leq \sum_{n=0}^{\infty}\left(\mathbb{E}_{\xi}\left|Y_{n+1}-Y_{n}\right|^{\alpha}\right)^{\frac{2}{\alpha}}$, we have $\mathbb{E}_{\xi} \mid \sum_{n=0}^{\infty}\left(Y_{n+1}-\right.$ $\left.Y_{n}\right)\left.^{2}\right|^{\frac{\alpha}{2}} \leq\left(\sum_{n=0}^{\infty}\left(\mathbb{E}_{\xi}\left|Y_{n+1}-Y_{n}\right|^{\alpha}\right)^{\frac{2}{\alpha}}\right)^{\frac{\alpha}{2}}$. Therefore, together with (4.1), we have

$$
\sup _{n} \mathbb{E}_{\xi}\left|Y_{n}-1\right|^{\alpha} \leq\left(\sum_{n=0}^{\infty}\left(\mathbb{E}_{\xi}\left|Y_{n+1}-Y_{n}\right|^{\alpha}\right)^{\frac{2}{\alpha}}\right)^{\frac{\alpha}{2}}
$$

So in order to prove $\sup _{n} \mathbb{E}_{\xi}\left|Y_{n}-1\right|<\infty$ a.s., we only need to prove that $\sum_{n=1}^{\infty}\left(\mathbb{E}_{\xi} \mid Y_{n+1}-\right.$ $\left.\left.Y_{n}\right|^{\alpha}\right)^{2 / \alpha}<\infty$ a.s.. By BDG-inequality and the convexity of $x^{\frac{\alpha}{2}}$, we get

$$
\begin{aligned}
\mathbb{E}_{\xi}\left|Y_{n+1}-Y_{n}\right|^{\alpha} & \leq C \mathbb{E}_{\xi}\left(\sum_{|u|=n} X_{u}^{2}\left(Y_{1}(u)-1\right)^{2}\right)^{\frac{\alpha}{2}}=C \mathbb{E}_{\xi}\left(\sum_{|u|=n} \frac{X_{u}^{2}}{Y_{n}(2)} Y_{n}(2)\left(Y_{1}(u)-1\right)^{2}\right)^{\frac{\alpha}{2}} \\
& \leq C \mathbb{E}_{\xi} \sum_{|u|=n} \frac{X_{u}^{2}}{Y_{n}(2)}\left(Y_{n}(2)\left(Y_{1}(u)-1\right)^{2}\right)^{\frac{\alpha}{2}}=C \mathbb{E}_{\xi} Y_{n}^{\frac{\alpha}{2}}(2) \mathbb{E}_{T^{n} \xi}\left|Y_{1}-1\right|^{\alpha} . \quad(4.3)
\end{aligned}
$$

Since $Y_{n}(2)=\sum_{|u|=n} X_{u}^{2} \leq D_{n}^{*} Y_{n}(1)$, we have

$$
\mathbb{E}_{\xi} Y_{n}^{\frac{\alpha}{2}}(2) \leq \mathbb{E}_{\xi} D_{n}^{* \frac{\alpha}{2}} Y_{n}^{\frac{\alpha}{2}} \leq\left(\mathbb{E}_{\xi} Y_{n}^{\alpha}\right)^{\frac{1}{2}}\left(\mathbb{E}_{\xi} D_{n}^{* \alpha}\right)^{\frac{1}{2}} \leq\left(\mathbb{E}_{\xi} Y_{n}^{\alpha}\right)^{\frac{1}{2}}\left(\mathbb{E}_{\xi} D_{0}^{\alpha} \cdots \mathbb{E}_{\xi} D_{n-1}^{\alpha}\right)^{\frac{1}{2}}
$$

By (4.2), (4.3) and (4.4), we have

$$
\begin{aligned}
\sup _{n} \mathbb{E}_{\xi} Y_{n}^{\alpha} & \leq \sup _{n} \mathbb{E}_{\xi}\left(\left|Y_{n}-1\right|+1\right)^{\alpha} \leq \sup _{n} 2^{\alpha}\left(\mathbb{E}_{\xi}\left|Y_{n}-1\right|^{\alpha}+1\right) \\
& \leq 2^{\alpha}\left(\sum_{n=0}^{\infty}\left(\mathbb{E}_{\xi} Y_{n}^{\frac{\alpha}{2}}(2) \mathbb{E}_{T^{n} \xi}\left|Y_{1}-1\right|^{\alpha}\right)^{\frac{2}{\alpha}}\right)^{\frac{\alpha}{2}}+2^{\alpha} \\
& \leq 2^{\alpha} \sup _{n}\left(\mathbb{E}_{\xi} Y_{n}^{\alpha}\right)^{\frac{1}{2}}\left[\sum_{n=0}^{\infty}\left(\mathbb{E}_{\xi} D_{0}^{\alpha} \cdots \mathbb{E}_{\xi} D_{n-1}^{\alpha}\right)^{\frac{1}{\alpha}}\left(\mathbb{E}_{T^{n} \xi}\left|Y_{1}-1\right|^{\alpha}\right)^{\frac{2}{\alpha}}\right]^{\frac{\alpha}{2}}+2^{\alpha} .
\end{aligned}
$$

Therefore,

$$
\sup _{n}\left(\mathbb{E}_{\xi} Y_{n}^{\alpha}\right)^{\frac{1}{2}} \leq 2^{\alpha}\left[\sum_{n=0}^{\infty}\left(\mathbb{E}_{\xi} D_{0}^{\alpha} \cdots \mathbb{E}_{\xi} D_{n-1}^{\alpha}\right)^{\frac{1}{\alpha}}\left(\mathbb{E}_{T^{n} \xi}\left|Y_{1}-1\right|^{\alpha}\right)^{\frac{2}{\alpha}}\right]^{\frac{\alpha}{2}}+2^{\alpha} / \inf _{n}\left(\mathbb{E}_{\xi} Y_{n}^{\alpha}\right)^{\frac{1}{2}}
$$

Since $\frac{1}{\alpha} \mathbb{E} \log \mathbb{E}_{\xi} D_{0}^{\alpha} \leq \frac{1}{\alpha} \mathbb{E} \log \rho_{\xi_{0}}(\alpha)<0$ and $\frac{2}{\alpha} \mathbb{E} \log \mathbb{E}_{\xi} Y_{1}^{\alpha}<\infty$, by Lemma 3.1, we see that

$$
\left[\sum_{n=0}^{\infty}\left(\mathbb{E}_{\xi} D_{0}^{\alpha} \cdots \mathbb{E}_{\xi} D_{n-1}^{\alpha}\right)^{\frac{1}{\alpha}}\left(\mathbb{E}_{T^{n} \xi}\left|Y_{1}-1\right|^{\alpha}\right)^{\frac{2}{\alpha}}\right]^{\frac{\alpha}{2}}<\infty \text { a.s.. }
$$


Notice that when $Y>0$, then $Y_{n}>0$ for all $n \geq n_{0}=n_{0}(\omega)$ large enough. Since $Z_{k}(\mathbb{R})=0$ implies $Z_{n}(\mathbb{R})=0$ for all $n>k$, it follows that $\inf _{n} Y_{n}>0$ a.s. on $\{Y>0\}$, so that $\inf _{n>0} Y_{n}^{\alpha}>0$ a.s. on $\{Y>0\}$. Since $\mathbb{P}(Y>0)>0$ implies that $\mathbb{P}_{\xi}(Y>0)>0$ a.s. $\left(\mathbb{P}_{\xi}(Y>0)\right.$ satisfies the $0-1$ law $)$, it follows that

$$
\inf _{n} \mathbb{E}_{\xi} Y_{n}^{\alpha}>0
$$

so that

$$
\frac{2^{\alpha}}{\inf _{n}\left(\mathbb{E}_{\xi} Y_{n}^{\alpha}\right)^{\frac{1}{2}}}<\infty \quad \text { a.s.. }
$$

From (4.5), (4.6) and (4.8), we obtain

$$
\sup _{n} \mathbb{E}_{\xi} Y_{n}^{\alpha}<\infty \text { a.s. }
$$

so that $\mathbb{E}_{\xi} Y^{\alpha}<\infty$ a.s..

We next prove part (ii). Assume that $\mathbb{E}_{\xi} Y^{\alpha}<\infty$. We only need to prove that $\mathbb{E} \log ^{+} \mathbb{E}_{\xi} \mid Y_{1}-$ $\left.1\right|^{\alpha}<\infty$. We divide the proof into two cases, according to $\alpha \geq 2$ and $1<\alpha<2$.

We first consider the case where $\alpha \geq 2$. By BDG-inequality and the convexity of $x^{\frac{\alpha}{2}}$, we obtain

$$
\begin{aligned}
\sup _{n} \mathbb{E}_{\xi}\left|Y_{n}-1\right|^{\alpha} & \geq C \mathbb{E}_{\xi}\left(\sum_{n=0}^{\infty}\left(Y_{n+1}-Y_{n}\right)^{2}\right)^{\frac{\alpha}{2}} \geq C \sum_{n=0}^{\infty} \mathbb{E}_{\xi}\left|Y_{n+1}-Y_{n}\right|^{\alpha} \\
& \geq C \sum_{n=0}^{\infty} \mathbb{E}_{\xi}\left|\sum_{|u|=n} X_{u}^{2}\left(Y_{1}(u)-1\right)^{2}\right|^{\frac{\alpha}{2}} \geq C \sum_{n=0}^{\infty} \mathbb{E}_{\xi} \sum_{|u|=n} X_{u}^{\alpha}\left|Y_{1}(u)-1\right|^{\alpha} \\
& =C \sum_{n=0}^{\infty} P_{n}(\alpha) \mathbb{E}_{T^{n} \xi}\left|Y_{1}-1\right|^{\alpha} .
\end{aligned}
$$

Therefore, since $\sup _{n} \mathbb{E}_{\xi}\left|Y_{n}-1\right|^{\alpha}<\infty$ a.s. and $\mathbb{E} \log \rho_{\xi_{0}}(\alpha)<0$, by Lemma 3.1, we have $\mathbb{E} \log ^{+} \mathbb{E}_{\xi}\left|Y_{1}-1\right|^{\alpha}<\infty$, which implies $\mathbb{E} \log ^{+} \mathbb{E}_{\xi} Y_{1}^{\alpha}<\infty$.

We next consider the case where $1<\alpha<2$. Let $\eta=\sum_{n} P_{n}(\alpha)$. By BDG-inequality and the concavity of $x^{\frac{\alpha}{2}}$, we obtain

$$
\begin{aligned}
\sup _{n} \mathbb{E}_{\xi}\left|Y_{n}-1\right|^{\alpha} & \geq C \mathbb{E}_{\xi}\left(\sum_{n=0}^{\infty}\left(Y_{n+1}-Y_{n}\right)^{2}\right)^{\frac{\alpha}{2}}=C \mathbb{E}_{\xi}\left(\sum_{n=0}^{\infty} \frac{P_{n}(\alpha)}{\eta} \frac{\eta}{P_{n}(\alpha)}\left(Y_{n+1}-Y_{n}\right)^{2}\right)^{\frac{\alpha}{2}} \\
& \geq C \mathbb{E}_{\xi} \sum_{n=0}^{\infty} \frac{P_{n}(\alpha)}{\eta} \frac{\eta^{\frac{\alpha}{2}}}{P_{n}^{\frac{\alpha}{2}}(\alpha)}\left|Y_{n+1}-Y_{n}\right|^{\alpha} \\
& =C \eta^{\frac{\alpha}{2}-1} \sum_{n=0}^{\infty} P_{n}^{1-\frac{\alpha}{2}}(\alpha) \mathbb{E}_{\xi}\left|Y_{n+1}-Y_{n}\right|^{\alpha}
\end{aligned}
$$

For the same reason, we have

$$
\begin{aligned}
\mathbb{E}_{\xi}\left|Y_{n+1}-Y_{n}\right|^{\alpha} & \geq C \mathbb{E}_{\xi}\left(\sum_{|u|=n} X_{u}^{2}\left|Y_{1}(u)-1\right|^{2}\right)^{\frac{\alpha}{2}}=C \mathbb{E}_{\xi}\left(\sum_{|u|=n} \frac{X_{u}^{2}}{Y_{n}(2)} Y_{n}(2)\left|Y_{1}(u)-1\right|^{2}\right)^{\frac{\alpha}{2}} \\
& \geq C \mathbb{E}_{\xi} \sum_{|u|=n} \frac{X_{u}^{2}}{Y_{n}(2)}\left(Y_{n}(2)\left|Y_{1}(u)-1\right|^{2}\right)^{\frac{\alpha}{2}}=C \mathbb{E}_{\xi} Y_{n}^{\frac{\alpha}{2}}(2) \mathbb{E}_{T^{n} \xi}\left|Y_{1}-1\right|^{\alpha} \\
& \geq C \inf _{n} \mathbb{E}_{\xi} Y_{n}^{\frac{\alpha}{2}}(2) \mathbb{E}_{T^{n} \xi}\left|Y_{1}-1\right|^{\alpha} .
\end{aligned}
$$


By (4.9) and (4.10), we have

$$
\sup _{n} \mathbb{E}_{\xi}\left|Y_{n}-1\right|^{\alpha} \geq C \eta^{\frac{\alpha}{2}-1} \inf _{n} \mathbb{E}_{\xi} Y_{n}^{\frac{\alpha}{2}}(2) \sum_{n=0}^{\infty} P_{n}^{1-\frac{\alpha}{2}}(\alpha) \mathbb{E}_{T^{n} \xi}\left|Y_{1}-1\right|^{\alpha}
$$

By (4.7), we have $\inf _{n} \mathbb{E}_{\xi} Y_{n}^{\alpha / 2}(2)>0$ a.s.. Since $\sup _{n} \mathbb{E}_{\xi}\left|Y_{n}-1\right|^{\alpha}<\infty$ and $\mathbb{E} \log \rho_{\xi_{0}}(\alpha)<0$, by Lemma 3.1, we obtain $\mathbb{E} \log ^{+} \mathbb{E}_{\xi}\left|Y_{1}-1\right|^{\alpha}<\infty$, which implies $\mathbb{E} \log ^{+} \mathbb{E}_{\xi} Y_{1}^{\alpha}<\infty$.

Finally, part (iii) follows directly from Lemma 3.1, part (2)(c).

\section{Proof of Theorem 2.4}

For $n \geq 0$, write $M_{n}=\sup _{|u|=n} X_{u}^{\beta-1}, \quad M=\sum_{n=1}^{\infty} M_{n}$.

Lemma 5.1 Let $\phi:[0, \infty) \rightarrow[0, \infty)$ be a convex and increasing function with $\phi(0)=0$ and $\phi(2 x) \leq c \phi(x)$ for some constant $c \in(0, \infty)$ and all $x>0$. Let $\beta \in(1,2]$. If the function $x \mapsto \phi\left(x^{1 / \beta}\right)$ is convex, then

$$
\begin{aligned}
\mathbb{E}_{\xi} \phi\left(\left|Y^{*}-1\right|\right) \leq C \sum_{n=1}^{\infty} & {\left[\mathbb{E}_{\xi}\left(\frac{M_{n-1}}{M} \phi\left(M^{1 / \beta} Y_{n-1}^{1 / \beta}\left(\mathbb{E}_{T^{n-1} \xi}\left|Y_{1}\right|^{\beta}\right)^{1 / \beta}\right)\right)\right.} \\
+ & \left.\mathbb{E}_{\xi} \sum_{|u|=n-1} \frac{X_{u}^{\beta}}{Y_{n-1}(\beta)} \phi\left(Y_{n-1}^{1 / \beta}(\beta)\left|Y_{1}(u)-1\right|\right)\right],
\end{aligned}
$$

where $C>0$ is a constant depending only on $c$ and $\beta$.

Proof By (3.6), we have

$$
\mathbb{E}_{\xi} \phi\left(Y^{*}-1\right) \leq C\left(\mathbb{E}_{\xi} \phi\left(\left(\sum_{n=1}^{\infty} \mathbb{E}_{\xi}\left(\left|Y_{n}-Y_{n-1}\right|^{\beta} \mid \mathcal{F}_{n-1}\right)\right)^{\frac{1}{\beta}}\right)+\sum_{n=1}^{\infty} \mathbb{E}_{\xi} \phi\left(\left|Y_{n}-Y_{n-1}\right|\right)\right)
$$

where $C>0$ is a constant only depending on $c$ and $\beta$. By BDG-inequality, the concavity of $x^{\frac{\beta}{2}}$ and the definition of $M_{n}$, we obtain

$$
\begin{aligned}
& \mathbb{E}_{\xi}\left(\left|Y_{n}-Y_{n-1}\right|^{\beta} \mid \mathcal{F}_{n-1}\right) \leq C \mathbb{E}_{\xi}\left(\left(\sum_{|u|=n-1} X_{u}^{2}\left(Y_{1}(u)-1\right)^{2}\right)^{\frac{\beta}{2}} \mid \mathcal{F}_{n-1}\right) \\
\leq & C \mathbb{E}_{\xi}\left(\sum_{|u|=n-1} X_{u}^{\beta}\left|Y_{1}(u)-1\right|^{\beta} \mid \mathcal{F}_{n-1}\right)=C \sum_{|u|=n-1} X_{u}^{\beta} \mathbb{E}_{T^{n-1} \xi}\left|Y_{1}-1\right|^{\beta} \\
\leq & C M_{n-1} Y_{n-1} \mathbb{E}_{T^{n-1} \xi}\left|Y_{1}-1\right|^{\beta} .
\end{aligned}
$$


where $Y_{1}(u)=\sum_{|v|=1} A_{u v}, \mathbb{P}_{\xi}\left(Y_{1}(u) \in \cdot\right)=\mathbb{P}_{T^{n-1} \xi}\left(Y_{1} \in \cdot\right)$ for $|u|=n-1$. By (5.3), using the fact that $\sum_{n=1}^{\infty} M_{n} M^{-1}=1$ and the convexity of $\phi\left(x^{\frac{1}{\beta}}\right)$, we have

$$
\begin{aligned}
& \mathbb{E}_{\xi} \phi\left(\left(\sum_{n=1}^{\infty} \mathbb{E}_{\xi}\left(\left|Y_{n}-Y_{n-1}\right|^{\beta} \mid \mathcal{F}_{n-1}\right)\right)^{\frac{1}{\beta}}\right) \\
\leq & \mathbb{E}_{\xi} \phi\left(\left(\sum_{n=1}^{\infty} C M_{n-1} Y_{n-1} \mathbb{E}_{T^{n-1} \xi}\left|Y_{1}-1\right|^{\beta}\right)^{\frac{1}{\beta}}\right) \\
= & \mathbb{E}_{\xi} \phi\left(\left(\sum_{n=1}^{\infty} C \frac{M_{n-1}}{M} M Y_{n-1} \mathbb{E}_{T^{n-1} \xi}\left|Y_{1}-1\right|^{\beta}\right)^{\frac{1}{\beta}}\right) \\
\leq & C \mathbb{E}_{\xi} \sum_{n=1}^{\infty} \frac{M_{n-1}}{M} \phi\left(M^{\frac{1}{\beta}} Y_{n-1}^{\frac{1}{\beta}}\left(\mathbb{E}_{T^{n-1} \xi}\left|Y_{1}-1\right|^{\beta}\right)^{\frac{1}{\beta}}\right) .
\end{aligned}
$$

By BDG-inequality and the convexity of $\phi\left(x^{\frac{1}{\beta}}\right)$, we obtain

$$
\begin{aligned}
& \mathbb{E}_{\xi} \phi\left(\left|Y_{n}-Y_{n-1}\right|\right) \leq C \mathbb{E}_{\xi} \phi\left(\left(\sum_{|u|=n-1} X_{u}^{\beta}\left|Y_{1}(u)-1\right|^{\beta}\right)^{\frac{1}{\beta}}\right) \\
= & C \mathbb{E}_{\xi} \phi\left(\left(\sum_{|u|=n-1} \frac{X_{u}^{\beta}}{Y_{n-1}(\beta)} Y_{n-1}(\beta)\left|Y_{1}(u)-1\right|^{\beta}\right)^{\frac{1}{\beta}}\right) \\
\leq & C \mathbb{E}_{\xi} \sum_{|u|=n-1} \frac{X_{u}^{\beta}}{Y_{n-1}(\beta)} \phi\left(Y_{n-1}^{\frac{1}{\beta}}(\beta)\left|Y_{1}(u)-1\right|\right) .
\end{aligned}
$$

By (5.2), (5.4) and (5.5), we obtain (5.1).

For the proof of Theorem 1.2, we will use the following lemma.

Lemma 5.2 Let $X$ be a non negative random variable, $l$ be a function slowly varying at $\infty$ and $\phi(x)=x^{\alpha} l(x)$ with $\alpha>1$. The following assertions are equivalent:

(i) $\mathbb{E} \log ^{+} \mathbb{E}_{\xi} \phi(X)<\infty$;

(ii) $\mathbb{E} \log ^{+} \mathbb{E}_{\xi} \phi(|X-c|)<\infty$, where $c>0$ is a constant.

Proof of Theorem 2.4 Let $\beta \in(1,2]$ and $\beta<\alpha$. Write $\phi(x)=x^{\alpha} l(x)$, we can assume that the functions $\phi$ and $x \mapsto \phi\left(x^{1 / \beta}\right)$ are convex on $[0, \infty)$, and $l(x)>0$ for all $x \geq 0$ (see [24]). Moreover, by choosing a smoothed version if necessary, we can suppose that $l$ is differentiable.

The equivalence between (ii) and (iii) is obvious following Theorem 2.1 in [32]. The rest of the proof is composed of the following two steps.

Step 1: prove that (i) implies (iii). Suppose that $\mathbb{E} \log ^{+} \mathbb{E}_{\xi} \phi\left(Y_{1}\right)<\infty$. By Lemma 5.1, we have

$$
\mathbb{E}_{\xi} \phi\left(Y^{*}-1\right) \leq C \sum_{n=1}^{\infty}\left(I_{1}(n)+I_{2}(n)\right),
$$


where

$$
\begin{aligned}
& I_{1}(n)=\mathbb{E}_{\xi} \frac{M_{n-1}}{M} \phi\left(M^{\frac{1}{\beta}} Y_{n-1}^{\frac{1}{\beta}}\left(\mathbb{E}_{T^{n-1} \xi}\left|Y_{1}-1\right|^{\beta}\right)^{\frac{1}{\beta}}\right), \\
& I_{2}(n)=\mathbb{E}_{\xi} \sum_{|u|=n-1} \frac{X_{u}^{\beta}}{Y_{n-1}(\beta)} \phi\left(Y_{n-1}^{\frac{1}{\beta}}(\beta)\left|Y_{1}(u)-1\right|\right) .
\end{aligned}
$$

Hence, in order to prove $\mathbb{E}_{\xi} \phi\left(\left|Y^{*}-1\right|\right)<\infty$ a.s., we only need to prove that $\sum_{n=1}^{\infty} I_{1}(n)<\infty$ a.s. and $\sum_{n=1}^{\infty} I_{2}(n)<\infty$ a.s..

We first prove that $\sum_{n=1}^{\infty} I_{1}(n)<\infty$ a.s.. Since $l$ is bounded away from 0 and $\infty$ on any compact subset of $[0, \infty)$, by Potter's theorem (see [12]), for $\varepsilon>0$, there exists $C=C(l, \varepsilon)>$ 1 such that $l(x) \leq C \max \left(x^{\varepsilon}, x^{-\varepsilon}\right)$ for all $x>0$. Therefore

$$
\begin{aligned}
\sum_{n=1}^{\infty} I_{1}(n) & =\sum_{n=1}^{\infty} \mathbb{E}_{\xi} M_{n-1} M^{\frac{\alpha}{\beta}-1} Y_{n-1}^{\frac{\alpha}{\beta}}\left(\mathbb{E}_{T^{n-1} \xi}\left|Y_{1}-1\right|^{\beta}\right)^{\frac{\alpha}{\beta}} l\left(M^{\frac{1}{\beta}} Y_{n-1}^{\frac{1}{\beta}}\left(\mathbb{E}_{T^{n-1} \xi}\left|Y_{1}-1\right|^{\beta}\right)^{\frac{1}{\beta}}\right) \\
& \leq C \sum_{n=1}^{\infty}\left(I_{1}^{+}(n)+I_{1}^{-}(n)\right),
\end{aligned}
$$

where

$$
\begin{aligned}
& I_{1}^{+}(n)=\mathbb{E}_{\xi} M_{n-1} M^{\frac{\alpha+\varepsilon}{\beta}-1} Y_{n-1}^{\frac{\alpha+\varepsilon}{\beta}}\left(\mathbb{E}_{T^{n-1} \xi}\left|Y_{1}-1\right|^{\beta}\right)^{\frac{\alpha+\varepsilon}{\beta}}, \\
& I_{1}^{-}(n)=\mathbb{E}_{\xi} M_{n-1} M^{\frac{\alpha-\varepsilon}{\beta}-1} Y_{n-1}^{\frac{\alpha-\varepsilon}{\beta}}\left(\mathbb{E}_{T^{n-1} \xi}\left|Y_{1}-1\right|^{\beta}\right)^{\frac{\alpha-\varepsilon}{\beta}} .
\end{aligned}
$$

Choose $\varepsilon_{1}>0$ and $\varepsilon_{2}>0$ small enough such that $\alpha-\varepsilon_{1}>\beta$ and $\frac{\left(\alpha+\varepsilon_{2}\right)\left(\alpha-\varepsilon_{2}\right)}{\alpha-\frac{(\beta+1) \varepsilon_{2}}{\beta-1}} \in(1, \alpha+\gamma)$, where $\gamma$ is defined below (see (5.9)). Let $\varepsilon=\min \left(\varepsilon_{1}, \varepsilon_{2}\right)$. Then $\alpha-\varepsilon>\beta$ and $\frac{(\alpha+\varepsilon)(\alpha-\varepsilon)}{\alpha-\frac{(\beta+1) \varepsilon}{\beta-1}} \in$ $(1, \alpha+\gamma)$. Using Hölder's inequality twice and Jensen's inequality, we obtain

$$
\begin{aligned}
& I_{1}^{+}(n) \leq\left(\mathbb{E}_{\xi}\left(M_{n-1} M^{\frac{\alpha+\varepsilon}{\beta}-1}\right)^{p_{1}}\right)^{\frac{1}{p_{1}}}\left(\mathbb{E}_{\xi} Y_{n-1}^{\frac{\alpha+\varepsilon}{\beta} p_{2}}\right)^{\frac{1}{p_{2}}}\left(\mathbb{E}_{T^{n-1} \xi}\left|Y_{1}-1\right|^{\beta}\right)^{\frac{\alpha+\varepsilon}{\beta}} \\
\leq & \left(\mathbb{E}_{\xi} M_{n-1}^{p_{1} q_{1}}\right)^{\frac{1}{p_{1} q_{1}}}\left(\mathbb{E}_{\xi} M^{\left(\frac{\alpha+\varepsilon}{\beta}-1\right) p_{1} q_{2}}\right)^{\frac{1}{p_{1} q_{2}}}\left(\mathbb{E}_{\xi} Y_{n-1}^{\frac{\alpha+\varepsilon}{\beta} p_{2}}\right)^{\frac{1}{p_{2}}}\left(\mathbb{E}_{T^{n-1} \xi}\left|Y_{1}-1\right|^{\beta}\right)^{\frac{\alpha+\varepsilon}{\beta}} \\
\leq & \left(\mathbb{E}_{\xi} Y_{n-1}^{\frac{\alpha+\varepsilon}{\beta} p_{2}}\right)^{\frac{1}{p_{2}}}\left(\mathbb{E}_{\xi} M_{n-1}^{p_{1} q_{1}}\right)^{\frac{1}{p_{1} q_{1}}}\left(\mathbb{E}_{\xi} M^{\left(\frac{\alpha+\varepsilon}{\beta}-1\right) p_{1} q_{2}}\right)^{\frac{1}{p_{1} q_{2}}}\left(\mathbb{E}_{T^{n-1} \xi}\left|Y_{1}-1\right|^{\alpha-\varepsilon}\right)^{\frac{\alpha+\varepsilon}{\alpha-\varepsilon}},
\end{aligned}
$$

where $p_{2}=\frac{\beta(\alpha-\varepsilon)}{\alpha+\varepsilon}, q_{1}=\frac{\alpha+\varepsilon}{\beta}$ and $\frac{1}{p_{1}}+\frac{1}{p_{2}}=\frac{1}{q_{1}}+\frac{1}{q_{2}}=1$. As $\mathbb{E} \log \rho_{\xi_{0}}(x)$ is convex for $\mathbb{E} \log \rho_{\xi_{0}}(1)=0$ and $\mathbb{E} \log \rho_{\xi_{0}}(\alpha)<0$, there exists some $\gamma \in(0,1)$ such that

$$
\mathbb{E} \log \rho_{\xi_{0}}(x)<0 \text { for } x \in(1, \alpha+\gamma]
$$

in particular,

$$
\mathbb{E} \log \rho_{\xi_{0}}(\alpha-\varepsilon)<0 \text { for } 0<\varepsilon<\alpha-1 \text {. }
$$

By Potter's theorem, for $\varepsilon>0$, there exists $C=C(l, \varepsilon)>0$ such that $l(x) \geq C x^{-\varepsilon}$ for all $x \geq 1$. Therefore $\mathbb{E}_{\xi}\left|Y_{1}-1\right|^{\alpha-\varepsilon} \leq 1+\frac{1}{C} \mathbb{E}_{\xi} \phi\left(\left|Y_{1}-1\right|\right)$ implies that

$$
\begin{aligned}
& \mathbb{E} \log ^{+} \mathbb{E}_{\xi}\left|Y_{1}-1\right|^{\alpha-\varepsilon} \leq \mathbb{E} \log ^{+} \mathbb{E}_{\xi}\left(1+\frac{1}{C} \mathbb{E}_{\xi} \phi\left(\left|Y_{1}-1\right|\right)\right) \\
\leq & 1+\log ^{+} \frac{1}{C}+\mathbb{E} \log ^{+} \mathbb{E}_{\xi}\left|Y_{1}-1\right|^{\alpha} l\left(Y_{1}-1\right)<\infty .
\end{aligned}
$$


By Theorem 2.3 together with (5.10) and (5.11), we know that

$$
\sup _{n \geq 1} \mathbb{E}_{\xi} Y_{n-1}^{\alpha-\varepsilon}<\infty \text { a.s.. }
$$

Since $p_{1} q_{1}(\beta-1)=\frac{(\alpha+\varepsilon)(\alpha-\varepsilon)}{\alpha-\frac{(\beta+1) \varepsilon}{\beta-1}} \in(1, \alpha+\gamma)$. we have $\mathbb{E} \log \rho_{\xi_{0}}\left(p_{1} q_{1}(\beta-1)\right)<0$. So by the triangular inequality for the norm \|\|$_{p_{1} q_{1}}$ in $L^{p_{1} q_{1}}$, we have

$$
\begin{aligned}
\left(\mathbb{E}_{\xi} M_{n}^{p_{1} q_{1}}\right)^{\frac{1}{p_{1} q_{1}}} & \leq\left(\mathbb{E}_{\xi}\left(\sum_{|u|=n} X_{u}^{\beta-1}\right)^{p_{1} q_{1}}\right)^{\frac{1}{p_{1} q_{1}}} \leq\left(\mathbb{E}_{\xi} \sum_{|u|=n} X_{u}^{(\beta-1) p_{1} q_{1}}\right)^{\frac{1}{p_{1} q_{1}}} \\
& =\left(P_{n-1}\left((\beta-1) p_{1} q_{1}\right)\right)^{\frac{1}{p_{1} q_{1}}}
\end{aligned}
$$

and

$$
\begin{aligned}
& \left(\mathbb{E}_{\xi} M^{\left(\frac{\alpha+\varepsilon}{\beta}-1\right) p_{1} q_{2}}\right)^{\frac{1}{p_{1} q_{2}}}=\left(\mathbb{E}_{\xi} M^{p_{1} q_{1}}\right)^{\frac{1}{p_{1} q_{2}}} \leq\left(\sum_{n=1}^{\infty}\left(\mathbb{E}_{\xi} M_{n-1}^{p_{1} q_{1}}\right)^{\frac{1}{p_{1} q_{1}}}\right)^{\frac{\alpha+\varepsilon}{\beta}-1} \\
\leq & \left(\sum_{n=1}^{\infty}\left(P_{n-1}\left((\beta-1) p_{1} q_{1}\right)\right)^{\frac{1}{p_{1} q_{1}}}\right)^{\frac{\alpha+\varepsilon}{\beta}-1}<\infty \text { a.s.. }
\end{aligned}
$$

By (5.8) and (5.13), we have

$$
\begin{aligned}
& \sum_{n=1}^{\infty} I_{1}^{+}(n) \leq \sup _{n \geq 1} \mathbb{E}_{\xi} Y_{n-1}^{\alpha-\varepsilon}\left(\mathbb{E}_{\xi} M^{\left(\frac{\alpha+\varepsilon}{\beta}-1\right) p_{1} q_{2}}\right)^{\frac{1}{p_{1} q_{2}}} \sum_{n=1}^{\infty}\left(\mathbb{E}_{\xi} M_{n-1}^{p_{1} q_{1}}\right)^{\frac{1}{p_{1} q_{1}}}\left(\mathbb{E}_{T^{n-1} \xi}\left|Y_{1}-1\right|^{\alpha-\varepsilon}\right)^{\frac{\alpha+\varepsilon}{\alpha-\varepsilon}} \\
\leq & \sup _{n \geq 1} \mathbb{E}_{\xi} Y_{n-1}^{\alpha-\varepsilon}\left(\mathbb{E}_{\xi} M^{\left(\frac{\alpha+\varepsilon}{\beta}-1\right) p_{1} q_{2}}\right)^{\frac{1}{p_{1} q_{2}}} \sum_{n=1}^{\infty}\left(P_{n-1}\left((\beta-1) p_{1} q_{1}\right)\right)^{\frac{1}{p_{1} q_{1}}}\left(\mathbb{E}_{T^{n-1} \xi}\left|Y_{1}-1\right|^{\alpha-\varepsilon}\right)^{\frac{\alpha+\varepsilon}{\alpha-\varepsilon}} .
\end{aligned}
$$

Therefore, since $\mathbb{E} \log \rho_{\xi_{0}}\left(p_{1} q_{1}(\beta-1)\right)<0$ and $\mathbb{E} \log ^{+} \mathbb{E}_{\xi} Y_{1}^{\alpha-\varepsilon}<\infty$, by Lemmas 3.1 and 5.2, together with (5.12) and (5.14), we have

$$
\sum_{n=1}^{\infty} I_{1}^{+}(n)<\infty \text { a.s.. }
$$

By an argument similar to that used above for the case of $I_{1}^{+}(n)$, choosing $\varepsilon>0$ small enough such that $\alpha-\varepsilon>\beta$, we have

$$
\begin{aligned}
& I_{1}^{-}(n) \leq\left(\mathbb{E}_{\xi} M_{n-1}^{p_{3}} M^{p_{3}\left(\frac{\alpha-\varepsilon}{\beta}-1\right)}\right)^{\frac{1}{p_{3}}}\left(\mathbb{E}_{\xi} Y_{n-1}^{p_{4} \frac{\alpha-\varepsilon}{\beta}}\right)^{\frac{1}{p_{4}}}\left(\mathbb{E}_{T^{n-1} \xi}\left|Y_{1}-1\right|^{\beta}\right)^{\frac{\alpha-\varepsilon}{\beta}} \\
\leq & \left(\mathbb{E}_{\xi} M_{n-1}^{p_{3} q_{3}}\right)^{\frac{1}{p_{3} q_{3}}}\left(\mathbb{E}_{\xi} M^{\left(\frac{\alpha-\varepsilon}{\beta}-1\right) p_{3} q_{4}}\right)^{\frac{1}{p_{3} q_{4}}}\left(\mathbb{E}_{\xi} Y_{n-1}^{\alpha-\varepsilon}\right)^{\frac{1}{p_{4}}} \mathbb{E}_{T^{n-1} \xi}\left|Y_{1}-1\right|^{\alpha-\varepsilon} \\
= & \left(\mathbb{E}_{\xi} M_{n-1}^{p_{3} q_{3}}\right)^{\frac{1}{p_{3} q_{3}}}\left(\mathbb{E}_{\xi} M^{p_{3} q_{3}}\right)^{\frac{1}{p_{3} q_{3}}\left(\frac{\alpha-\varepsilon}{\beta}-1\right)}\left(\mathbb{E}_{\xi} Y_{n-1}^{\alpha-\varepsilon}\right)^{\frac{1}{p_{4}}} \mathbb{E}_{T^{n-1} \xi}\left|Y_{1}-1\right|^{\alpha-\varepsilon} \\
\leq & C\left(P_{n-1}\left((\beta-1) p_{3} q_{3}\right)\right)^{\frac{1}{p_{3} q_{3}}}\left(\mathbb{E}_{\xi} Y_{n-1}^{\alpha-\varepsilon}\right)^{\frac{1}{p_{4}}} \mathbb{E}_{T^{n-1} \xi}\left|Y_{1}-1\right|^{\alpha-\varepsilon} \\
= & C\left(P_{n-1}(\alpha-\varepsilon)\right)^{\frac{1}{p_{3} q_{3}}}\left(\mathbb{E}_{\xi} Y_{n-1}^{\alpha-\varepsilon}\right)^{\frac{1}{p_{4}}} \mathbb{E}_{T^{n-1} \xi}\left|Y_{1}-1\right|^{\alpha-\varepsilon},
\end{aligned}
$$


where $q_{3}=(\alpha-\varepsilon) / \beta>1, p_{3}=\frac{\beta}{\beta-1}>1, \frac{1}{p_{3}}+\frac{1}{p_{4}}=\frac{1}{q_{3}}+\frac{1}{q_{4}}=1$. Therefore

$$
\begin{aligned}
\sum_{n=1}^{\infty} I_{1}^{-}(n) & \leq C \sum_{n=1}^{\infty}\left(P_{n-1}(\alpha-\varepsilon)\right)^{\frac{1}{p_{3} q_{3}}}\left(\mathbb{E}_{\xi} Y_{n-1}^{\alpha-\varepsilon}\right)^{\frac{1}{p_{4}}} \mathbb{E}_{T^{n-1} \xi}\left|Y_{1}-1\right|^{\alpha-\varepsilon} \\
& \leq C \sup _{n}\left(\mathbb{E}_{\xi} Y_{n-1}^{\alpha-\varepsilon}\right)^{\frac{1}{p_{4}}} \sum_{n=1}^{\infty}\left(P_{n-1}(\alpha-\varepsilon)\right)^{\frac{1}{p_{3} q_{3}}} \mathbb{E}_{T^{n-1} \xi}\left|Y_{1}-1\right|^{\alpha-\varepsilon}
\end{aligned}
$$

Hence, since $\mathbb{E} \log \rho_{\xi_{0}}(\alpha-\varepsilon)<0$ and $\mathbb{E} \log ^{+} \mathbb{E}_{\xi} Y_{1}^{\alpha-\varepsilon}<\infty$, by Lemmas 3.1 and 5.2 together with (5.12), we have

$$
\sum_{n=1}^{\infty} I_{1}^{-}(n)<\infty \text { a.s.. }
$$

By (5.7), (5.15) and (5.17), we obtain

$$
\sum_{n=1}^{\infty} I_{1}(n)<\infty \text { a.s.. }
$$

We next prove $\sum_{n=1}^{\infty} I_{2}(n)<\infty$ a.s.. By Potter's theorem, for $\varepsilon>0$, there exists $C=C(l, \varepsilon)>0$ such that $l(x y) \leq C l(x) \max \left\{y^{\varepsilon}, y^{-\varepsilon}\right\}$. Hence, we obtain

$$
\begin{aligned}
I_{2}(n) & =C \mathbb{E}_{\xi} \sum_{|u|=n-1} \frac{X_{u}^{\beta}}{Y_{n-1}(\beta)} Y_{n-1}^{\frac{\alpha}{\beta}}(\beta)\left|Y_{1}(u)-1\right|^{\alpha} l\left(Y_{n-1}^{\frac{1}{\beta}}(\beta)\left|Y_{1}(u)-1\right|\right) \\
& \leq C\left(I_{2}^{+}(n)+I_{2}^{-}(n)\right) .
\end{aligned}
$$

where

$$
\begin{aligned}
& I_{2}^{+}(n)=\mathbb{E}_{\xi} Y_{n-1}^{\frac{\alpha+\varepsilon}{\beta}}(\beta) \mathbb{E}_{T^{n-1} \xi} \phi\left(\left|Y_{1}-1\right|\right), \\
& I_{2}^{-}(n)=\mathbb{E}_{\xi} Y_{n-1}^{\frac{\alpha-\varepsilon}{\beta}}(\beta) \mathbb{E}_{T^{n-1} \xi} \phi\left(\left|Y_{1}-1\right|\right) .
\end{aligned}
$$

In order to prove $\sum_{n=1}^{\infty} I_{2}(n)<\infty$ a.s., we only need to prove that $\sum_{n=1}^{\infty} I_{2}^{+}(n)<\infty$ a.s. and $\sum_{n=1}^{\infty} I_{2}^{-}(n)<\infty$ a.s.. We now consider $\sum_{n=1}^{\infty} I_{2}^{+}(n)$. By $M_{n}=\sup _{|u|=n} X_{u}^{\beta-1}$ and Höler's inequality, we have

$$
I_{2}^{+}(n) \leq \mathbb{E}_{\xi}\left(M_{n-1} Y_{n-1}\right)^{\frac{\alpha+\varepsilon}{\beta}} \mathbb{E}_{T^{n-1} \xi} \phi\left(\left|Y_{1}-1\right|\right) \leq\left(\mathbb{E}_{\xi} M_{n-1}^{\frac{\alpha+\varepsilon}{\beta} p}\right)^{\frac{1}{p}}\left(\mathbb{E}_{\xi} Y_{n-1}^{\frac{\alpha+\varepsilon}{\beta} p^{*}}\right)^{\frac{1}{p^{*}}} \mathbb{E}_{T^{n-1} \xi} \phi\left(\left|Y_{1}-1\right|\right),
$$

where $p^{*}=\frac{\beta(\alpha-\varepsilon)}{\alpha+\varepsilon}>1, \quad \frac{1}{p}+\frac{1}{p^{*}}=1$. Therefore, we have

$$
\begin{aligned}
& \sum_{n=1}^{\infty} I_{2}^{+}(n) \leq C \sum_{n=1}^{\infty}\left(\mathbb{E}_{\xi} M_{n-1}^{\frac{\alpha+\varepsilon}{\beta} p}\right)^{\frac{1}{p}}\left(\mathbb{E}_{\xi} Y_{n-1}^{\frac{\alpha+\varepsilon}{\beta} p^{*}}\right)^{\frac{1}{p^{*}}} \mathbb{E}_{T^{n-1} \xi} \phi\left(\left|Y_{1}-1\right|\right) \\
\leq & C \sum_{n=1}^{\infty}\left(\mathbb{E}_{\xi} \sum_{|u|=n-1} X_{u}^{(\beta-1) \frac{\alpha+\varepsilon}{\beta} p}\right)^{\frac{1}{p}}\left(\mathbb{E}_{\xi} Y_{n-1}^{\frac{\alpha+\varepsilon}{\beta} p^{*}}\right)^{\frac{1}{p^{*}}} \mathbb{E}_{T^{n-1} \xi} \phi\left(\left|Y_{1}-1\right|\right) \\
\leq & C \sup _{n \geq 1}\left(\mathbb{E}_{\xi} Y_{n-1}^{\alpha-\varepsilon}\right)^{\frac{1}{p^{*}}} \sum_{n=1}^{\infty}\left(P_{n-1}(\tilde{p})\right)^{\frac{1}{p}} \mathbb{E}_{T^{n-1} \xi} \phi\left(\left|Y_{1}-1\right|\right) .
\end{aligned}
$$


where $\varepsilon>0$ is small enough such that $\tilde{p}:=\frac{\alpha+\varepsilon}{\beta}(\beta-1) p \in(1, \alpha+\gamma)$, then $\mathbb{E} \log m_{0}(\tilde{p})<0$. Since $\mathbb{E} \log \rho_{\xi_{0}}(\tilde{p})<0$ and $\mathbb{E} \log ^{+} \mathbb{E}_{\xi} \phi\left(\left|Y_{1}-1\right|\right)<\infty$, by Lemma 3.1 and the above display, we have

$$
\sum_{n=1}^{\infty} I_{2}^{+}(n)<\infty \text { a.s.. }
$$

We now consider $\sum_{n=1}^{\infty} I_{2}^{-}(n)$. Similar to the case of $I_{2}^{+}(n)$, we have

$$
\begin{aligned}
I_{2}^{-}(n) & =\mathbb{E}_{\xi}\left(Y_{n-1}(\beta)\right)^{\frac{\alpha-\varepsilon}{\beta}} \mathbb{E}_{T^{n-1} \xi} \phi\left(\left|Y_{1}-1\right|\right) \leq \mathbb{E}_{\xi}\left(M_{n-1} Y_{n-1}\right)^{\frac{\alpha-\varepsilon}{\beta}} \mathbb{E}_{T^{n-1} \xi} \phi\left(\left|Y_{1}-1\right|\right) \\
& \leq\left(\mathbb{E}_{\xi} M_{n-1}^{\frac{\alpha-\varepsilon}{\beta} q}\right)^{\frac{1}{q}}\left(\mathbb{E}_{\xi} Y_{n-1}^{\frac{\alpha-\varepsilon}{\beta} q^{*}}\right)^{\frac{1}{q^{*}}} \mathbb{E}_{T^{n-1} \xi} \phi\left(\left|Y_{1}-1\right|\right)
\end{aligned}
$$

where $q^{*}=\beta, q=\frac{\beta}{\beta-1}$ and $\varepsilon \in(0, \alpha-1)$. Therefore, we have

$$
\begin{aligned}
\sum_{n=1}^{\infty} I_{2}^{-}(n) & \leq \sum_{n=1}^{\infty}\left(\mathbb{E}_{\xi} M_{n-1}^{\frac{\alpha-\varepsilon}{\beta} q}\right)^{\frac{1}{q}}\left(\mathbb{E}_{\xi} Y_{n-1}^{q^{*} \frac{\alpha-\varepsilon}{\beta}}\right)^{\frac{1}{q^{*}}} \mathbb{E}_{T^{n-1} \xi} \phi\left(\left|Y_{1}-1\right|\right) \\
& \leq \sup _{n \geq 1}\left(\mathbb{E}_{\xi} Y_{n-1}^{\alpha-\varepsilon}\right)^{\frac{1}{q^{*}}} \sum_{n=1}^{\infty}\left(\mathbb{E}_{\xi} M_{n-1}^{\frac{\alpha-\varepsilon}{\beta} q}\right)^{\frac{1}{q}} \mathbb{E}_{T^{n-1} \xi} \phi\left(\left|Y_{1}-1\right|\right) \\
& \leq \sup _{n \geq 1}\left(\mathbb{E}_{\xi} Y_{n-1}^{\alpha-\varepsilon}\right)^{\frac{1}{q^{*}}} \sum_{n=1}^{\infty}\left(P_{n-1}(\alpha-\varepsilon)\right)^{\frac{1}{q}} \mathbb{E}_{T^{n-1} \xi} \phi\left(\left|Y_{1}-1\right|\right) .
\end{aligned}
$$

Therefore, since $\mathbb{E} \log \rho_{\xi_{0}}(\alpha-\varepsilon)<0$ and $\mathbb{E} \log ^{+} \mathbb{E}_{\xi} \phi\left(\left|Y_{1}-1\right|\right)<\infty$, by Lemma 3.1, we have

$$
\sum_{n=1}^{\infty} I_{2}^{-}(n)<\infty \text { a.s.. }
$$

By (5.19), (5.20) and (5.21), we get

$$
\sum_{n=1}^{\infty} I_{2}(n)<\infty \text { a.s.. }
$$

Combining (5.6), (5.18) and (5.22), we obtain $\mathbb{E}_{\xi} \phi\left(\left|Y^{*}-1\right|\right)<\infty$ a.s. which is equivalent to $\mathbb{E}_{\xi} \phi\left(Y^{*}\right)<\infty$ a.s..

Step 2: prove that (iii) implies (i). Assume that $\mathbb{E}_{\xi} \phi\left(Y^{*}\right)<\infty$ a.s.. We only need to prove that $\mathbb{E} \log ^{+} \mathbb{E}_{\xi} \phi\left(\left|Y_{1}-1\right|\right)<\infty$ by Lemma 5.2. We divide the proof into two cases, according to $\alpha>2$ and $1<\alpha \leq 2$.

(a) We first consider the case $\alpha>2$. By Lemma 3.2(ii) and the convexity of $\phi_{1 / 2}(x)=$ $\phi\left(x^{1 / 2}\right)$, we obtain

$$
\begin{aligned}
& \mathbb{E}_{\xi} \phi\left(\left|Y^{*}-1\right|\right) \geq A \mathbb{E}_{\xi} \phi\left(\left(\sum_{n=1}^{\infty}\left(Y_{n}-Y_{n-1}\right)^{2}\right)^{\frac{1}{2}}\right) \\
& \geq A \sum_{n=1}^{\infty} \mathbb{E}_{\xi} \phi\left(\left(\left(Y_{n}-Y_{n-1}\right)^{2}\right)^{\frac{1}{2}}\right)=A \sum_{n=1}^{\infty} \mathbb{E}_{\xi} \phi\left(\left|Y_{n}-Y_{n-1}\right|\right) .
\end{aligned}
$$


For the same reason, we have

$$
\mathbb{E}_{\xi} \phi\left(\left|Y_{n}-Y_{n-1}\right|\right) \geq A \mathbb{E}_{\xi} \phi\left(\left(\left|\sum_{|u|=n-1} X_{u}^{2}\left(Y_{1}(u)-1\right)^{2}\right|\right)^{\frac{1}{2}}\right) \geq A \mathbb{E}_{\xi}\left(\sum_{|u|=n-1} \phi\left(X_{u}\left|Y_{1}(u)-1\right|\right)\right)
$$

Choose $\varepsilon>0$ small enough such that $\alpha-\varepsilon>1$. By Potter's theorem, there exists $C=C(l, \varepsilon)$ such that $l(x y) \geq C l(x) \min \left\{y^{\varepsilon}, y^{-\varepsilon}\right\}$, for all $x>0$ and $y>0$. Hence, we have

$$
\begin{aligned}
& \mathbb{E}_{\xi} \phi\left(\left|Y^{*}-1\right|\right) \geq A \sum_{n=1}^{\infty} \mathbb{E}_{\xi}\left(\sum_{|u|=n-1} \phi\left(X_{u}\left|Y_{1}(u)-1\right|\right)\right) \\
\geq & A C \sum_{n=1}^{\infty} \mathbb{E}_{\xi}\left(\sum_{|u|=n-1} X_{u}^{\alpha}\left|Y_{1}(u)-1\right|^{\alpha} \min \left(X_{u}^{\varepsilon}, X_{u}^{-\varepsilon}\right) l\left(\left|Y_{1}(u)-1\right|\right)\right) \\
= & A C \sum_{n=1}^{\infty} \mathbb{E}_{\xi} \sum_{|u|=n-1} X_{u}^{\alpha} \min \left(X_{u}^{-\varepsilon}, X_{u}^{\varepsilon}\right) \mathbb{E}_{T^{n-1} \xi} \phi\left(\left|Y_{1}-1\right|\right) \\
= & A C \sum_{n=1}^{\infty} \min \left(P_{n-1}(\alpha-\varepsilon), P_{n-1}(\alpha+\varepsilon)\right) \mathbb{E}_{T^{n-1} \xi} \phi\left(\left|Y_{1}-1\right|\right) .
\end{aligned}
$$

Since $-\infty<\mathbb{E} \log \rho_{\xi_{0}}(\alpha)<0$ for $\alpha>1$, by the law of large numbers, we have for $\delta>0$ and $n>n(\xi)$ large enough,

$$
P_{n-1}(\alpha-\varepsilon)>e^{\left(\mathbb{E} \log \rho_{\xi_{0}}(\alpha-\varepsilon)-\delta\right)(n-1)}, P_{n-1}(\alpha+\varepsilon)>e^{\left(\mathbb{E} \log \rho_{\xi_{0}}(\alpha+\varepsilon)-\delta\right)(n-1)},
$$

then there is $a(\alpha, \varepsilon) \in(-\infty, 0)$ such that a.s. $P_{n-1}(\alpha-\varepsilon) \geq e^{a(\alpha, \varepsilon)(n-1)}$ and $P_{n-1}(\alpha+\varepsilon) \geq$ $e^{a(\alpha, \varepsilon)(n-1)}$ for $n>n(\xi)$ large enough. Therefore the preceding lower bound of $\mathbb{E} \phi\left(Y^{*}-1\right)$ implies that

$$
\sum_{n=1}^{\infty} e^{a(\alpha, \varepsilon)(n-1)} \mathbb{E}_{T^{n-1} \xi} \phi\left(\left|Y_{1}-1\right|\right)<\infty \quad \text { a.s.. }
$$

Hence, by Lemma 3.1 we obtain $\mathbb{E} \log ^{+} \mathbb{E}_{\xi} \phi\left(\left|Y_{1}-1\right|\right)<\infty$ which, by Lemma 5.2, implies $\mathbb{E} \log ^{+} \mathbb{E}_{\xi} \phi\left(Y_{1}\right)<\infty$.

(b) We next consider the case where $1<\alpha \leq 2$. Let $F=\sum_{n=1}^{\infty} P_{n-1}(\alpha)$. By BDGinequality and the concavity of $\phi_{1 / 2}(x)=\phi\left(x^{1 / 2}\right)$, we have

$$
\begin{aligned}
\mathbb{E}_{\xi} \phi\left(\left|Y^{*}-1\right|\right) & \geq C \mathbb{E}_{\xi} \phi\left(\left(\sum_{n=1}^{\infty}\left(Y_{n}-Y_{n-1}\right)^{2}\right)^{\frac{1}{2}}\right)=C \mathbb{E}_{\xi} \phi_{\frac{1}{2}}\left(\sum_{n=1}^{\infty}\left(Y_{n}-Y_{n-1}\right)^{2}\right) \\
& =C \mathbb{E}_{\xi} \phi_{\frac{1}{2}}\left(\left(\sum_{n=1}^{\infty} \frac{P_{n-1}(\alpha)}{F} \frac{F}{P_{n-1}(\alpha)}\left(Y_{n}-Y_{n-1}\right)^{2}\right)\right) \\
& \geq C \mathbb{E}_{\xi} \sum_{n=1}^{\infty} \frac{P_{n-1}(\alpha)}{F} \phi_{\frac{1}{2}}\left(\frac{F}{P_{n-1}(\alpha)}\left(Y_{n}-Y_{n-1}\right)^{2}\right) \\
& =C \sum_{n=1}^{\infty} \frac{P_{n-1}(\alpha)}{F} \mathbb{E}_{\xi} \phi\left(F^{\frac{1}{2}} P_{n-1}^{-\frac{1}{2}}(\alpha)\left|Y_{n}-Y_{n-1}\right|\right) .
\end{aligned}
$$


By BDG-inequality and the concavity of $\phi_{1 / 2}(x)$, we get

$$
\begin{aligned}
& \mathbb{E}_{\xi} \phi\left(F^{\frac{1}{\alpha}} P_{n-1}^{-\frac{1}{2}}(2)\left|Y_{n}-Y_{n-1}\right|\right) \geq C \mathbb{E}_{\xi} \phi\left(\left(\sum_{|u|=n-1} F \frac{1}{P_{n-1}(\alpha)} X_{u}^{2}\left|Y_{1}(u)-1\right|^{2}\right)^{\frac{1}{2}}\right) \\
= & C \mathbb{E}_{\xi} \phi_{\frac{1}{2}}\left(\sum_{|u|=n-1} F \frac{1}{P_{n-1}(\alpha)} \frac{X_{u}^{2}}{Y_{n-1}(2)} Y_{n-1}(2)\left|Y_{1}(u)-1\right|^{2}\right) \\
\geq & C \mathbb{E}_{\xi} \sum_{|u|=n-1} \frac{X_{u}^{2}}{Y_{n-1}(2)} \phi\left(\frac{F^{\frac{1}{2}}}{P_{n-1}^{\frac{1}{2}}(\alpha)} Y_{n-1}^{\frac{1}{2}}(2)\left|Y_{1}(u)-1\right|\right) .
\end{aligned}
$$

By Potter's theorem, for $\varepsilon>0$, there exists $A=A(l, \varepsilon)>0$ such that $l(x y) \geq A l(y) \min \left\{x^{\varepsilon}, x^{-\varepsilon}\right\}$.

Therefore

$$
\begin{aligned}
& \mathbb{E}_{\xi} \phi\left(\left|Y^{*}-1\right|\right) \\
\geq & \frac{A C}{F} \sum_{n=1}^{\infty} P_{n-1}(\alpha) \mathbb{E}_{\xi} \sum_{|u|=n-1} \frac{X_{u}^{2}}{Y_{n-1}(2)} \min \left(F^{\frac{\alpha+\varepsilon}{2}} Y_{n-1}^{\frac{\alpha+\varepsilon}{2}}(2) P_{n-1}^{\frac{\alpha+\varepsilon}{2}}(\alpha), F^{\frac{\alpha-\varepsilon}{2}} Y_{n-1}^{\frac{\alpha-\varepsilon}{2}}(2) P_{n-1}^{\frac{\alpha-\varepsilon}{2}}(\alpha)\right) \phi\left(\left|Y_{1}(u)-1\right|\right) \\
= & \frac{A C}{F} \sum_{n=1}^{\infty} P_{n-1}(\alpha) \min \left(F^{\frac{\alpha+\varepsilon}{2}} \mathbb{E}_{\xi} Y_{n-1}^{\frac{\alpha+\varepsilon}{2}}(2), F^{\frac{\alpha-\varepsilon}{2}} \mathbb{E}_{\xi} Y_{n-1}^{\frac{\alpha-\varepsilon}{2}}(2)\right) \min \left(P_{n-1}^{\frac{\alpha+\varepsilon}{2}}(\alpha), P_{n-1}^{\frac{\alpha-\varepsilon}{2}}(\alpha)\right) \mathbb{E}_{T^{n-1} \xi} \phi\left(\left|Y_{1}-1\right|\right) \\
\geq & \frac{A C}{F} \mathbb{E}_{\xi} U \sum_{n=1}^{\infty} \min \left(P_{n-1}^{1+\frac{\alpha+\varepsilon}{2}}(\alpha), P_{n-1}^{1+\frac{\alpha-\varepsilon}{2}}(\alpha)\right) \mathbb{E}_{T^{n-1} \xi} \phi\left(\left|Y_{1}-1\right|\right),
\end{aligned}
$$

where $U=\min \left(F^{\frac{\alpha+\varepsilon}{2}} \inf _{n} Y_{n-1}^{\frac{\alpha+\varepsilon}{2}}(2), F^{\frac{\alpha-\varepsilon}{2}} \inf _{n} Y_{n-1}^{\frac{\alpha-\varepsilon}{2}}(2)\right)$. By (4.9), we have $\inf _{n>0} Y_{n}(2)>0$ a.s.. Hence $U>0$ a.s. on $\{Y>0\}$. Since $\mathbb{P}_{\xi}(Y>0)>0$, this implies that $\mathbb{E}_{\xi} U>0$. Since $\infty<\mathbb{E} \log \rho_{\xi_{0}}(\alpha)<0$, by the law of large numbers, there is $a \in(\infty, 0)$ such that a.s. $P_{n-1}(\alpha) \geq e^{(n-1) a}$ for $n \geq n(\xi)$ large enough. Therefore the preceding lower bound of $\mathbb{E}_{\xi} \phi\left(Y^{*}-1\right)$ implies that

$$
\sum_{n=1}^{\infty} e^{(n-1) a\left(1+\frac{\alpha+\varepsilon}{2}\right)} \mathbb{E}_{T^{n-1} \xi} \phi\left(\left|Y_{1}-1\right|\right)<\infty \text { a.s.. }
$$

Hence, by Lemma 3.1, we get $\mathbb{E} \log ^{+} \mathbb{E}_{\xi} \phi\left(\left|Y_{1}-1\right|\right)<\infty$ which, by Lemma 5.2, implies that $\mathbb{E} \log ^{+} \mathbb{E}_{\xi} \phi\left(Y_{1}\right)<\infty$. Thus we have proved that (iii) implies (i).

\section{Acknowledgements}

The work has benefited from the support of the French government Investissements dAvenir program ANR-11-LABX-0020-01. It has been partially supported by the National Natural Science Foundation of China (Grants no. 11571052, no. 11401590, no. 11731012 and no. 11671404 ), and by Hunan Natural Science Foundation (China, grant no. 2017JJ2271).

\section{References}

[1] K. B. Athreya and S. Karlin. On branching processes in random environments. I: Extinction probabilities. Ann. Math. Statist. 42 (1971a) 1499-1520. 
[2] K. B. Athreya and S. Karlin. On branching processes in random environments. II: Limit theorems. Ann. Math. Statist. 42 (1971b) 1843-1858.

[3] K. B. Athreya and P. E. Ney. Branching processes (Berlin: Springer, 1972).

[4] G. Alsmeyer and U. Rösler. On the existence of $\phi$-moments of the limit of a normalized supercritical Galton-Watson process. J. Theor. Probab. 17 (2004) 905-928.

[5] G. Alsmeyer and D. Kuhlbusch. Double martingale structure and existence of $\phi$-moments for weighted branching processes. Münster J. Math. 3 (2010) 163-212.

[6] J. Barral. Generalized vector multiplicative cascades. Adv. Appl. Prob. 33 (2001) 874895.

[7] J. D. Biggins. Martingale convergence in the branching random walk. J. Appl. Prob. 14 (1977), no.1, 25-37.

[8] J. D. Biggins. Uniform convergence of martingale in the branching random walk. Ann. Prob. 20(1) (1992), 137-151.

[9] J. D. Biggins. A. E. Kyprianou. Measure change in multitype branching. Adv. in Appl. Probab. 36 (2) (2004) 544-581.

[10] N. H. Bingham and R. A. Doney. Asymptotic properties of supercritical branching processes. I: The Galton- Watson process. Adv. Appl. Prob. 6 (1974) 711-731.

[11] N. H. Bingham and R. A. Doney. Asymptotic properties of supercritical branching processes. II: Crump-Mode and Jirina process. Adv. Appl. Prob. 7 (1975) 66-82.

[12] K. H. Bingham, C. M. Goldie, and J. L. Teugels. Regular Variation. Cambridge Univ: Press, Cambridge, 1987.

[13] X. Chen, H. He. On large deviation probabilities for empirical distribution of branching random walks: Schröder case and Böttcher case.

[14] Y. S. Chow and H. Teicher. Probability Theory: Independence, Interchangeability, Martingales. New York, Springer, 1995.

[15] A. K. Grintsevichyus. On the continuity of the distribution of a sum of dependent variables connected with independent walks on lines. Theory Prob. Appl. 19 (1974) 163-168.

[16] Y. Guivarc'h and Q. Liu. Proprietes asympotiques des processus de branchement en environnement aleatoire. C. R. Acad. Sci. Paris, Ser I. 332 (2001) 339-344.

[17] C. Huang. Limit theorems and the convergence rate of some branching processes and branching random walk. Ph.D. Thesis, Universit de Bretagne-Sud (France) 2010.

[18] C. Huang and Q. Liu. Convergence in $L^{p}$ and its exponential rate for a branching process in a random environment. Electron. J. Prob. 104(19) (2014) 1-22. 
[19] Y. Hu and Z. Shi. Minimal position and critical martingale convergence in branching random walks, and directed polymers on disordered trees. Ann. Probab., 37 (2009) 742789 .

[20] D. Kuhlbusch. On weighted branching process in random environment. Stoch. Prob. Appl. 109 (2004) no.1, 113-144.

[21] Y. Li and Q. Liu. Age-dependent Branching processes in random environments. Sci China Ser A. 51(10) (2008) 1807-1830.

[22] X. Liang. Asymptotic properties of the Mandelbrot's Martingale and the branching random walks. Ph.D. Thesis, Universit de Bretagne-Sud (France) 2010.

[23] X. Liang and Q. Liu. Weighted moments for the limit of a normalized supercritical Galton-Watson process. C. R. Acad. Sci. Paris, Ser. I. 351 (2013a) 769-773.

[24] X. Liang and Q. Liu. Weighted moments of the limit of a branching process in a random environment. Proc. Steklov Inst. Math. 282 (2013b) 127-145.

[25] X. Liang and Q. Liu. Weighted moments for Mandelbrot's martingales. Electron. Commun. Probab. 20 (85) (2015) 1-12.

[26] Q. Liu. On generalized multiplicascades. Stoc. Proc. Appl. 86 (2000) 263-286.

[27] Q. Liu. Branching random walks in random environment. Proceedings of the 4th International Congress of Chinese Mathematicians (ICCM 2007), Vol. II, 702-219. Eds:L.Ji, K.Liu, L.Yang, S.-T. Yau. 2007.

[28] Z. Gao, Q. Liu. Exact convergence rates in central limit theorems for a branching random walk with a random environment in time. Stoch. Proc. Appl. 126 (2016) 2634-2664.

[29] Z. Gao, Q. Liu, H. Wang. Central limit theorems for a branching random walk with a random environment in time. Acta Mathematica Scientia 34(2)(2014) 501-512.

[30] B. Mandelbrot. Multiplications aléatoires et distributions invaricantes par moyenne pondérée aléatoire. C. R. Acad. Sci. Pairs 287 (1974) 289-292,355-358.

[31] Z. Shi. Branching random walks, in École d'Été de Probabilités de Saint-Flour XLII C 2012. Lecture Notes in Mathematics, vol. 2151, Springer, Berlin, 2015.

[32] Y. Wang, Z. Liu, Q. Liu, Y. Li. Quenched weighted moments of a supercritical branching process in a random environment. Preprint. 